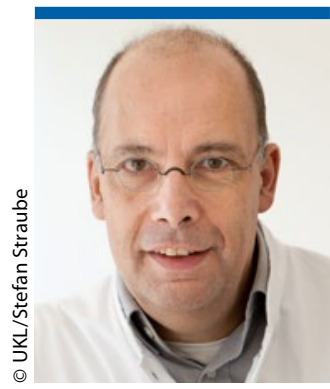

Dr. rer. nat. Roberto Frontini

Direktor der Klinikapotheke, Zentrum für Arzneimittelsicherheit in Leipzig

\title{
Nachgefragt
}

\section{„Biosimilars sind besser untersucht als Generika“}

? Biosimilars sind nicht identisch zu den Referenzpräparaten. Worin besteht der Unterschied?

Roberto Frontini: Biosimilars sind Proteine und immer glykosyliert. Der hauptsächliche Unterschied zum Original besteht in der Länge und der Position der Zucker. Aber darin unterscheiden sich auch verschiedene Chargen der Originale. Die Glykosylierung lässt sich nicht vollständig standardisieren - im Gegensatz zur Struktur der Proteine, die man sehr gut definieren kann und die identisch ist. Die Variabilität, die zwischen Biosimilar und Original erlaubt ist, ist die Variabilität, die auch innerhalb der Originale stattfindet; so steht es in einem Positionspapier der EMA.

? Werden Wirksamkeit und Sicherheit der Biosimilars durch die Behörden ausreichend sichergestellt? Frontini: Ja, ich denke zu 100\%. Wir lassen Generika zu allein aufgrund von In-vitro-Daten zur Bioverfügbarkeit. Für Biosimilars werden dagegen klinische Studien mit direktem Vergleich zum Original verlangt, bei dem das Outcome im selben Bereich liegen muss. Ich glaube, dass Biosimilars besser untersucht sind als Generika.

? Wie kritisch sehen Sie die Extrapolation auf andere als die getestete Indikation?

Frontini: Das ist durchaus ein kritischer Punkt. Aber die EMA hat dafür strenge Regeln aufgestellt. Und wenn man es nüchtern naturwissenschaftlich betrachtet, muss ein Wirkstoff an einem Rezeptor andocken und dort wirken. Wenn derselbe Wirkmechanismus auch bei einer anderen Krankheit relevant ist, ist der Wirksamkeitsnachweis fast zu $100 \%$ extrapolierbar, davon bin ich überzeugt.

? Es gibt Bedenken, dass Biosimilars unerwartete Immunreaktionen auslösen könnten. Zu Recht?

Frontini: Bei dieser Frage war ich vor Jahren vorsichtiger, weil gerade die Zuckerketten für Immunreaktionen wichtig sind. Inzwischen haben wir zum Beispiel mit Filgrastim-Nachahmerprodukten zehn Jahre Erfahrung und intensive Pharmakovigilanz. Die Daten zeigen, dass es mit den Biosimilars nicht mehr Antikörper und nicht mehr Immunreaktionen gibt. Ich bin daher mittlerweile auch in diesem Punkt weniger vorsichtig geworden.

? Was müssen Ärzte bei der Verordnung von Biosimilars beachten?

Frontini: Niemand sollte jetzt nur den Substanznamen auf ein Rezept schreiben, sondern aus Pharmakovigilanzgründen den Produktnamen. Außerdem sollte die Chargennummer dokumentiert werden - damit man verfolgen kann, ob es Unterschiede zum Original gibt.
? Die Umstellung von einem Originalpräparat auf ein Biosimilar ist umstritten. Zu Unrecht?

Frontini: Hier muss man ein bisschen unterscheiden. Bei Filgrastim zum Beispiel können wir von heute auf morgen auf ein anderes Produkt wechseln und es passiert gar nichts. Für komplexere Wirkstoffe gibt es noch nicht so viele Daten, aber ich bin überzeugt, dass es aus wissenschaftlicher Sicht möglich ist, einen Austausch vorzunehmen. Aber das sollte die gemeinsame Entscheidung von Arzt und Patient und Apotheker sein. Man muss intensiv mit den Patienten sprechen und sie aufklären. Wenn der Patient ein Problem damit hat, auch wenn es irrational ist, kann das Noceboeffekte auslösen.

? Leisten Biosimilars einen Beitrag zu einer besseren Versorgung?

Frontini: Absolut. Schließlich muss persönlich auch jeder auf sein Budget achten, und wer vernünftig wirtschaftet, kann mehr einkaufen. Genau das muss man auch den Patienten vermitteln. Wir wollen keine Billigmedizin, aber eine preisgünstige Medizin, die uns erlaubt, Interventionen zu machen, für die sonst das Geld nicht da wäre.

? Sie begrüßen also Zielvereinbarungen für Biosimilars?

Frontini: Die Quotenregelung verstehe ich nicht: Wenn ein Präparat gleichwertig, aber preiswerter ist, dann ist jede Quote falsch. Dann gibt es keinen Grund, Neueinstellungen mit einem anderen Präparat zu machen. In einer idealen Welt würde ich ab morgen alle neuen Patienten auf Biosimilars einstellen und versuchen, Patienten mit Dauertherapien davon zu überzeugen, dass ein Switch nicht gefährlich ist. Ich würde aber keinen Wechsel vornehmen, wenn bei den Patienten ein innerer Widerstand da ist.

? Bisher sind Biosimilars für acht Referenzprodukte und ein relativ eingeschränktes Indikationsspektrum verfügbar. Wie wird das in zehn Jahren aussehen?

Frontini: Ich denke, es wird ähnlich sein wie heute mit den Generika; die sind in den 1980er-Jahren auch verteufelt worden. In zehn Jahren werden wir aus wirtschaftlichen Gründen einen Markt haben, der vor allem aus Biosimilars besteht. Daran kommen wir nicht vorbei. Das System hat begrenzte Ressourcen und wir müssen den Umgang damit optimieren, wenn wir allen Patienten helfen wollen.

Das Interview führte Dr. Beate Schumacher. 